\title{
Metodología para el desarrollo de aplicaciones móviles
}

\section{Methodology for mobile application development}

\section{Maira Cecilia Gasca Mantilla}

Ingeniero Electrónico, Magíster en Mantenimiento Industrial. Docente de la Universidad Antonio Nariño. Santa Marta, Colombia.

Contacto:magasca@uan.edu.co

Luis Leonardo Camargo Ariza

Ingeniero Electrónico, Magíster en Ingeniería Electrónica. Docente de la Universidad del Magdalena. Santa Marta, Colombia.

Contacto:lcamargoa@unimagdalena.edu.co

\section{Byron Medina Delgado}

Ingeniero Electrónico, Magíster en Ingeniería Electrónica. Docente de la Universidad Francisco de Paula Santander. Cúcuta, Colombia.

Contacto: byronmedina@ufps.edu.co

Fecha de recepción: 1 de marzo de 2013

Clasificación del artículo: investigación

Fecha de aceptación: 27 de agosto de 2013

Financiamiento: Universidad del Magdalena

Palabras clave: comunicación móvil; servicio de información; software de código abierto.

Key words: Information Services; Mobile Communication; Open Source Software.

\section{RESUMEN}

El artículo presenta la evolución de los servicios de telefonía móvil en Latinoamérica contextualizados en las diferentes generaciones tecnológicas, las características del software para dispositivos móviles y una propuesta de método de trabajo para el desarrollo de aplicaciones para móviles. El método se basa en la conceptualización de las tecnologías y las metodologías ágiles para el desarrollo de software, y su objetivo principal es facilitar la creación de nuevas aplicaciones y servicios exitosos. El método se desar- rolla en cinco etapas: etapa de análisis, donde se obtienen y clasifican los requerimientos y se personaliza el servicio; etapa de diseño, momento en el que se define el escenario tecnológico y se estructura la solución por medio de algún diagrama o esquema, integrando tiempos y recursos; etapa de desarrollo, cuando se implementa el diseño en un producto de software; etapa de prueba de funcionamiento, donde se emula y simula el producto ajustando detalles, se instala en equipos reales y se evalúa el rendimiento, y posteriormente se evalúa el potencial de éxito; y finalmente, en la etapa de entrega, se define el canal de distribución 


\section{investigación}

de la aplicación, con el propósito de adecuar la aplicación al mismo. Además, el artículo presenta los resultados del desarrollo de un servicio msalud para Android y J2ME utilizando el método propuesto, el servicio está dirigido a pacientes que requieren o deseen tener un control periódico de las medidas corporales de tensión arterial y glucosa, servicio que obtuvo un potencial de éxito en los usuarios de prueba.

\section{ABSTRACT}

This paper presents: the evolution of mobile telephony services in Latin America contextualized in different technology generations, the features of software for mobile devices, and proposes a method for developing mobile applications in Latin America. This method is based on the conceptualization of contemporary telecommunications technologies and the agile software development methodologies. The main objective of

\section{INTRODUCCIÓN}

En la última década, el uso de la telefonía móvil en Latinoamérica ha tenido un creciente auge; la disminución de los costos en los equipos y la evolución de las tecnologías móviles han permitido aumentar el número de usuarios y las velocidades de transferencia de información, garantizando la penetración en el mercado con un promedio de 103 líneas telefónicas por cada 100 habitantes en el 2011 en Latinoamérica (UIT, 2012).

Un valor agregado que le permitió a la telefonía móvil una alta penetración en el mercado y un grado de aceptación alto por parte de los usuarios, es el poder ofrecer servicios alternos a los canales de voz.

La primera generación $1 \mathrm{G}$ de telefonía móvil, por ser de naturaleza analógica, solo podía prestar this method is to facilitate the creation and evaluation of new applications and services. The method is developed in five steps: the analysis stage, the requirements is obtained and classified and the service is customize; the design stage, in this stage is defined the technological and the solution is structured by some chart or diagram; the development stage, the design is implemented in a software product; the performance test stage, the application is emulates and simulates to adjusting details in the product, the software is installs in real equipment and is evaluated the performance and success; the delivery stage, the distribution channel of the application is defined, in order to adapt the application at the same. Furthermore, the paper presents the results of developing a mhealth service in Android and J2ME using the proposed method, service for patients who need or wish to have regular monitoring of measurements of blood pressure and glucose. The service has a satisfactory evaluation with test users.

el servicio de voz. La segunda generación es el comienzo de la era digital en la interfaz de radio y los operadores pudieron prestar servicios de transferencia de datos; fue en ese momento cuando se popularizó el Servicio de Mensajería Corta, Short Message Service (SMS). En redes de 2G como: Sistema Global para las Comunicaciones Móviles, Global System for Mobile Communications (GSM) y Servicio Avanzado de Telefonía Móvil-Digital, Digital-Advanced Mobile Phone Service (D-AMPS).

Con la implementación de tecnologías como: Servicio General de Paquetes de Radio, General Packet Radio Service (GPRS), Tasa de Datos Mejorada para la Evolución de GSM, Enhanced Data Rates GSM of Evolution (EDGE) y Acceso Múltiple por División de Código, Code Division Multiple Access (CDMA IS95, IS95B); se dio paso a la generación $2.5 \mathrm{G}$, aumentando la velocidad

Metodología para el desarrollo de aplicaciones móviles Maira Cecilia Gasca Mantilla / Luis Leonardo Camargo Ariza / Byron Medina Delgado 


\section{investigación}

de transferencia (Halonen, Romero y Melero, 2003), y con esto aparecieron nuevos servicios como el acceso a Internet desde el móvil por medio del Protocolo para Aplicaciones Inalámbricas, Wireless Application Protocol (WAP), el Servicio de Mensajería Mejorada, Enhanced Messaging Service (EMS), el Servicio de Mensajería Multimedia, Multimedia Messaging Service (MMS), entre otros (Montilla, 2004).

En la tercera generación $3 \mathrm{G}$ y $3.5 \mathrm{G}$ aparecen nuevos servicios, entre los que se destacan la videoconferencia y el Sistema de Posicionamiento Global, Global Positioning System (GPS), además del uso de las redes del operador de telefonía para acceder a Internet desde un computador con altas velocidades de descarga, High Speed Downlink Packet Access (HSDPA) (Holma y Toskala, 2004).

La cuarta generación $4 \mathrm{G}$ con tecnologías como: Long Term Evolution (LTE), LTE Avanzado, LTE Advanced (LTE-A), High Speed Packet Access (HSPA+) y el estándar IEEE 802.16; ofrecen servicios basados completamente en el Protocolo de Internet (IP), con velocidades de transferencia hasta de 100 Mbps y con Calidad de Servicios, Quality of Service (QoS) (Agusti et al., 2010).

Paralelamente al crecimiento de las redes, la evolución de los teléfonos móviles ha conllevado a la integración de diversas tecnologías a estos dispositivos, tecnologías como WiFi, Bluetooth, GPS, infrarrojo, touchscreen, USB, entre otras. Esto ha permitido que el teléfono celular sea compatible con una amplia gama de dispositivos y pueda sincronizarse con otros equipos para el intercambio de información.

La disponibilidad de las mejoras tecnológicas, tanto en las redes de los operadores como en los dispositivos móviles, están creando en las personas la demanda del uso de nuevos servicios. Servicios que se basan en estas tecnologías para solucionar problemas específicos en el ámbito empresarial (m-business), comercial (m-commer$c e$ ), académico (m-learning), de salud ( $m$-health) y social (redes sociales como: correo electrónico instantáneo de Blackberry, Facebook, Twitter, MySpace, Skype, entre otros) (Autelsi, 2009).

Los servicios móviles representan las capacidades de comunicación que los operadores ponen a disposición de los clientes "Usuarios". Estas capacidades están completamente definidas a través de protocolos y funciones estandarizadas, al menos en su forma de uso.

En la actualidad, la mayoría de los servicios móviles están desarrollados en: HTML 5, WAP, Java 2 Micro Edición (J2ME), C\#, Silverlight, .NET, entre otros. También, en aplicaciones nativas para los Sistemas Operativos de los móviles (S.O. del móvil), como: Android, Symbian, iOS y MeeGo.

El escenario tecnológico actual posiciona a los desarrolladores de servicios como la nueva fuerza de negocios en las redes de próxima generación Application Stores, allí radica la importancia de unificar metodologías y entornos para el desarrollo de servicios, que faciliten la creación de estos con alto nivel de calidad, llevando dicho desarrollo al éxito, de forma atractiva y eficiente.

El desarrollo de aplicaciones para proveer servicios móviles, difiere del desarrollo de software tradicional en muchos aspectos, lo que provoca que las metodologías usadas para estos entornos móviles, también difieran de las del software clásico (Rahimian y Ramsin, 2008).

Las características especiales de los entornos móviles como el canal de radio, la capacidad de los terminales, la portabilidad, el tiempo de salida al mercado "Time-to-Market", la movilidad del usuario, entre otras; exigen nuevas tendencias para desarrollar el software móvil en Latinoamérica. 


\section{investigación}

- Las condiciones del canal de radio en las comunicaciones móviles, como el ancho de banda variable, la disponibilidad de la red en el área del servicio, la diversidad en los estándares de la interfaz de aire, la dependencia de la tecnología implantada por el operador; diferencian los entornos fijos alámbricos e inalámbricos de los entornos móviles.

- Las capacidades de los terminales, especificaciones como el tamaño de la pantalla, el tiempo útil de la batería, los teclados reducidos, la capacidad de procesamiento y almacenamiento, las tecnologías alternas incluidas en el terminal, entre otros; determinan los recursos disponibles de hardware de la aplicación.

- La total portabilidad de las aplicaciones se dificulta por la diversidad técnica en el software y el hardware de los equipos en los que se ejecuta la aplicación móvil.

- El tiempo de salida al mercado refleja una industria cambiante tecnológicamente, donde los servicios están condicionados, generalmente, por el tiempo que se tomen los desarrolladores para transformar una idea o una solicitud, en un producto o solución acabada y lista para ser usada y facturada.

- La movilidad y la localización, son variables que determinan el uso del servicio desde cualquier lugar y en cualquier instante, características relacionadas directamente a los entornos y servicios móviles.

Las metodologías ágiles para el desarrollo de software, surgieron como una solución inmediata, garantizando la realización de proyectos en corto plazo, las cuales se pueden ajustar a los Time-toMarker de los servicios móviles, como es el caso de las metodologías Mobile-D (Kynkäänniemi y Komulainen, 2006), y Rapid7 (Kylmäkoski, 2005). Sin embargo, no garantizan el potencial de éxito de estos, éxito que depende en gran parte del conocimiento electrónico y de telecomunica- ciones, del entorno específico donde se ejecuta la aplicación (terminales y redes), además de la caracterización del cliente latinoamericano.

Por las razones anteriores, se propone la "Metodología para el Desarrollo de Aplicaciones Móviles, MDAM", metodología usada para la creación de un servicio m-salud (mHealth) denominado DrMovil y que es el caso de uso.

El término $m$ Health se define como la unión de la computación móvil, sensores médicos y tecnologías de comunicación, para el cuidado de la salud (Istepanian, Jovanov y Zhang, 2004). MHealth es una propuesta tecnológica que en los últimos años ha surgido como un segmento importante de la telemedicina y su objetivo principal es mejorar los servicios de salud, integrando los beneficios de movilidad y ubicuidad, propios de los sistemas móviles, a los tratamientos de cuidados de la salud tradicional, tratando de llevar la atención de salud a la gente y no la gente al sistema de salud. Las aplicaciones de mHealth están creando mecanismos para el intercambio de información relacionada con el cuidado de la salud, incluso en lugares remotos y de escasos recursos, debido a la gran área de cobertura e influencia social de las redes de telefonía móvil, convirtiéndose en un factor estratégico para salvar vidas (Vital Wave Consulting, 2009).

\section{METODOLOGÍA}

La metodología propuesta para el desarrollo de aplicaciones para móviles se fundamenta en la experiencia de investigaciones previas en aplicaciones móviles, la evaluación del potencial de éxito para servicios de tercera generación denominada $6 \mathrm{M}$, la ingeniería de software educativo con modelado orientado por objetos (ISE-OO), y principalmente en los valores de las metodologías ágiles. 


\section{investigación}

De la ISE-OO se hereda el enfoque de los micromundos interactivos y la orientación por objetos; los elementos de los micromundos más utilizados en los servicios móviles interactivos son: Mundo, Escenarios, Personajes y Roles, Argumento e Historia, Variables Compensatorias, Variables de Control, Variables de Resultado, Zonas de Comunicación, Ambientación-Caracterización, Recuperación de Estados Anteriores, Manejo de Información del Usuario, Mecanismos para Análisis de Desempeño, Ampliación de las Posibilidades del Micromundo, Personalización del Ambiente y, Soporte a la Comunicación en Grupo, entre otros (Gómez, Galvis y Mariño, 1998).

De las metodologías ágiles se heredan los conceptos inmersos en los cuatro postulados o manifiesto ágil (Beck et al., 2001).

- Desarrollar software que funciona más que conseguir buena documentación.

- La respuesta ante el cambio es más importante que el seguimiento de un plan.
- Colaboración con el cliente sobre negociación contractual.

- Individuos e interacciones sobre procesos y herramientas.

De la 6 M's se extrae la concepción de que las aplicaciones móviles deben garantizar el cumplimiento de las necesidades de los usuarios y al mismo tiempo generen ingresos. La $6 \mathrm{M}$ 's debe su nombre a los seis atributos que se miden para evaluar el éxito del servicio propuesto: Movement (Movimiento), Moment (Momento), Me (Yo), Multi-user (Multiusuario), Money (Dinero) y $\mathrm{Ma}$ chines (Máquinas) (Ahonen, Barret y Golding, 2002).

La metodología se encuentra enmarcada en cinco fases como se muestra en la figura 1, denominadas: análisis, diseño, desarrollo, pruebas de funcionamiento y entrega. A continuación se describe cada una de las actividades que intervienen en el desarrollo de la propuesta.

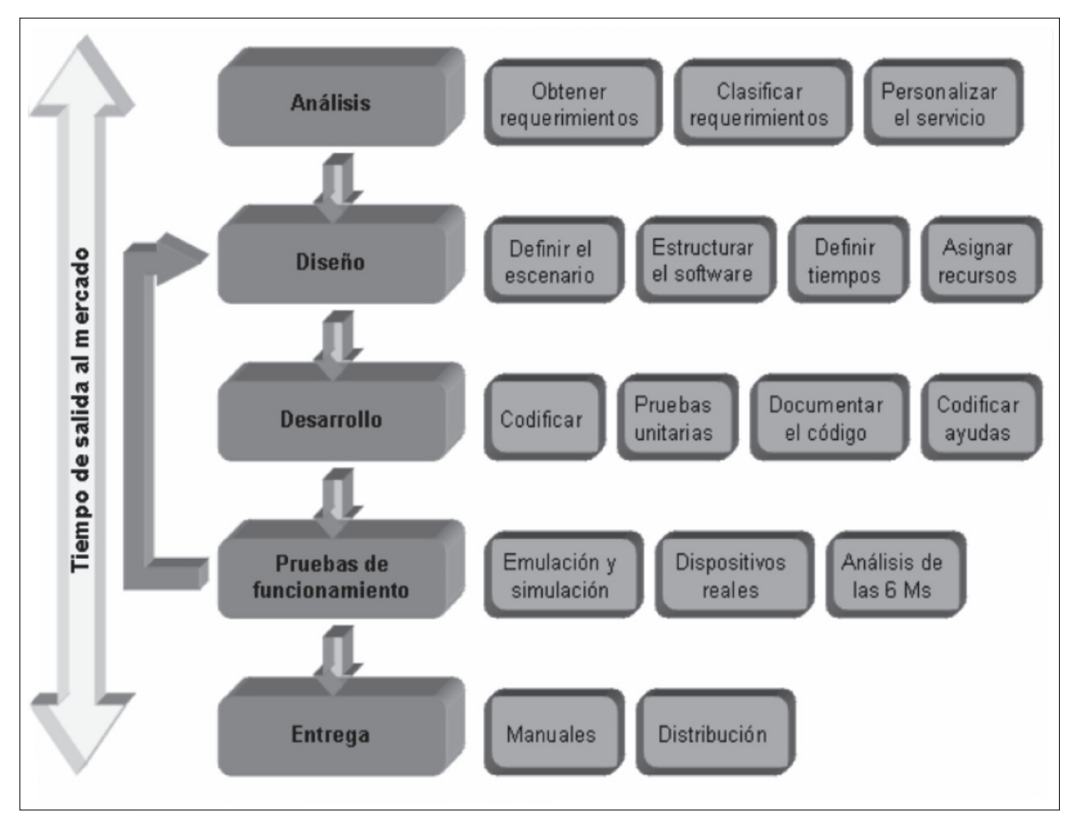

Figura 1. Etapas de la metodología para el desarrollo de aplicaciones móviles

Fuente: elaboración propia. 


\section{investigación}

\section{Análisis}

En esta fase se analizan las peticiones o requerimientos de las personas o entidad para la cual se desarrolla el servicio móvil "Cliente", el propósito es definir las características del mundo o entorno de la aplicación. Se realizan tres tareas: obtener requerimientos, clasificar los requerimientos y personalizar el servicio.

- Obtener requerimientos: se sugiere hacer una serie de entrevistas al cliente, para que manifieste los síntomas del problema o necesidades que se pretenden solucionar con las tecnologías móviles, o simplemente, para que señale las características que debe tener la aplicación.

- Clasificar los requerimientos: una vez identificados los requerimientos que debe tener el software, se procede a clasificarlos. Dichos requerimientos se pueden clasificar en entorno, mundo, funcionales y no funcionales.

El entorno se refiere a todo lo que rodea al servicio. Por ejemplo, las características técnicas del dispositivo móvil del cliente, el sistema operativo subyacente (móvil y servidores), la tecnología utilizada para la transferencia de información, el Sistema Manejador de Base de Datos, Data Base Management System (DBMS), si se requiere, el formato de archivos y, otros módulos tecnológicos utilizados para el servicio.

El mundo es la forma cómo interactúan el usuario y la aplicación. Aquí se encuentran los requerimientos de la Interfaz Gráfica de Usuario, Graphical User Interface (IGU), la forma en que el software va a generar los datos de salida, el formato de los datos y los demás requerimientos que involucren la comunicación hombre-máquina, considerando la gama tecnológica de los teléfonos móviles de los usuarios a la que va dirigida el servicio.
Los requerimientos funcionales son todos aquellos que demandan una función dentro del sistema. Se deben definir claramente cada una de las tareas que debe realizar la aplicación.

Los requerimientos no funcionales son la estabilidad, la portabilidad, el rendimiento, el tiempo de salida al mercado y, el costo, entre otros.

- Personalizar el servicio: adicionalmente se deben analizar aspectos de la cotidianidad del cliente como preferencias, costumbres y particularidades del usuario, con el propósito de garantizar la aceptación del servicio.

\section{Diseño}

El objetivo de esta etapa es plasmar el pensamiento de la solución mediante diagramas o esquemas, considerando la mejor alternativa al integrar aspectos técnicos, funcionales, sociales y económicos. A esta fase se retorna si no se obtiene lo deseado en la etapa prueba de funcionamiento.

Se realizan cuatro actividades en esta fase: definir el escenario, estructurar el software, definir tiempos y asignar recursos.

- Definir el escenario: las aplicaciones móviles se pueden diseñar para ejecutarse en diferentes escenarios, dependiendo del sistema de conexión y sincronización con el servidor o aplicación central; el proceso de sincronización se realiza para insertar, modificar o borrar información. Entre los diferentes escenarios se encuentran los siguientes: 1) desconectado: los procesos se realizan en el dispositivo móvil desconectado, después de terminar el proceso, si se requiere, puede conectarse con una aplicación central mediante el proceso de sincronización. 2) Semiconectado: los procesos pueden ejecutarse en el dispositivo móvil desconectado, pero se requiere establecer conexión en algún momento para terminar el proceso, al sincronizar la información 


\section{investigación}

con el servidor o aplicación central. En los escenarios desconectado y semiconectado se recomienda utilizar los protocolos y tecnologías que se ajusten al servicio y capacidades tecnológicas del dispositivo. Algunos son: Media Transfer Protocol (MTP), Near Field Communication (NFC), SlowSync, FastSync, SyncML, entre otros. 3) Conectado: el dispositivo debe estar siempre conectado con la aplicación central o servidor para su correcto funcionamiento, no se almacenan datos o archivos en el móvil, la sincronización se realiza mediante la validación de formularios, usualmente se utiliza el Protocolo de Transferencia de Hipertexto (Hypertext Transfer Protocol, HTTP).

- Estructurar el software: se deben utilizar algunos diagramas de Modelado de Lenguaje Unificado, Unified Modeling Language (UML), según las necesidades del proyecto, modelando el sistema desde varias perspectivas, ver figura 2.

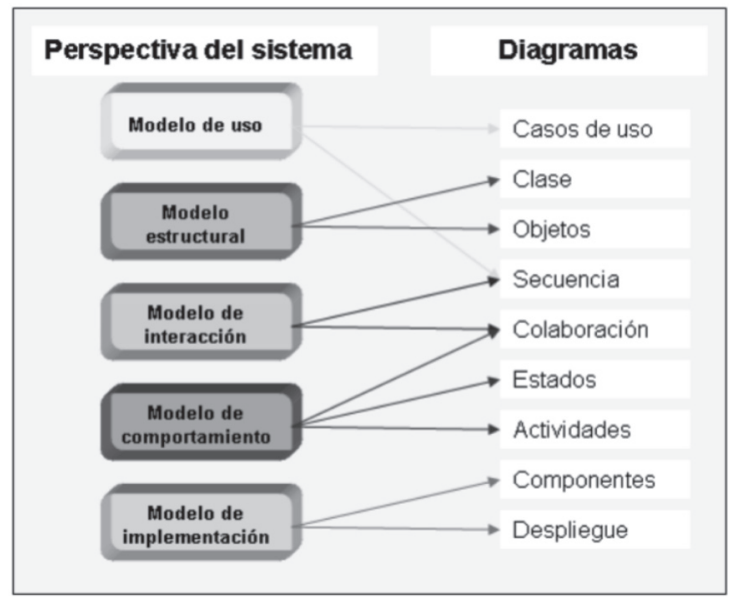

Figura 2. Posibles diagramas para el desarrollo de aplicaciones móviles

Fuente: elaboración propia.

Se sugiere traducir los requerimientos obtenidos de la etapa anterior en un diagrama que describa en forma objetiva el servicio por implementar. Además, definir un patrón de diseño para flexibi- lizar, modular y reutilizar lo desarrollado; la selección del patrón de diseño debe estar acorde con el escenario del servicio. Algunos patrones que se ajustan a los escenarios de las aplicaciones móviles son: modelo vista de controlador, diseño de capas, entre otros.

- Definir tiempos: se establecen los plazos para cada una de las actividades restantes, con el objetivo de terminar la aplicación a tiempo para su salida al mercado. Se debe tener en cuenta el diseño computacional del software realizado en la tarea anterior y, las características volátiles y dinámicas de los servicios móviles.

- Asignar recursos: se asignan los recursos para realizar cada actividad y alcanzar los objetivos propuestos, se deben considerar recursos humanos, financieros y tecnológicos. Además, se deben seleccionar las herramientas para el desarrollo de la aplicación móvil.

\section{Desarrollo}

El objetivo de esta fase es implementar el diseño en un producto de software. En esta etapa se realizan las siguientes actividades:

- Codificar: se escribe en el lenguaje de programación seleccionado, cada una de las partes definidas en los diagramas realizados en la etapa de diseño.

- Pruebas unitarias: se verifica el funcionamiento de la aplicación. En primer lugar, se comprueba la correcta operación de cada elemento desarrollado - objeto, clase, actividad, documento, entre otros - en forma individual; posteriormente, se pone en funcionamiento el conjunto de elementos, comprobando la interrelación entre ellos. Se ejecuta y se observan los resultados obtenidos, para compararlos con los esperados. 
- Documentar el código: a medida que se codifica y se prueba cada elemento, se redacta la pequeña documentación sobre lo desarrollado.

- Codificar ayudas: además del manual de instalación y de usuario, deben existir una serie de ayudas que informen de manera didáctica lo que puede hacer el usuario con la aplicación, estas ayudas deben ser codificadas en el mismo lenguaje de programación e integrada en la interfaz de aplicación para visualizarlas en el móvil.

\section{Pruebas de funcionamiento}

El objetivo de esta fase es verificar el funcionamiento de la aplicación en diferentes escenarios y condiciones; para esto se realizan las siguientes tareas:

- Emulación y simulación: se realizan pruebas simulando el escenario y emulando el dispositivo móvil, explorando todas las utilidades y funciones de la aplicación, introduciendo diferentes datos, inclusive erróneos, para medir la funcionalidad y el nivel de robustez del software. Si se encuentran algunas fallas, se debe regresar a la etapa de codificación en la fase de desarrollo para solucionar los problemas, si las pruebas son satisfactorias se procede a la etapa de pruebas con dispositivos reales.

- Dispositivos reales: deben hacerse pruebas de campo en equipos reales para medir el desempeño y el rendimiento del aplicativo. Si se encuentran fallas en el tiempo de ejecución, si el software no cumple con los requerimientos especificados, o si el cliente solicita un cambio de última hora, hay que regresar a la fase de diseño para reestructurar y solucionar el inconveniente presentado.

- Análisis de las 6 M's: para valorar el potencial de éxito del servicio, se sugiere buscar un grupo de expertos en el campo del desa-

\section{investigación}

rrollo móvil para que utilicen el método de evaluación de las 6 M's, y califiquen la presencia de los seis atributos en la aplicación desarrollada.

Cualquier servicio que brinde un gran valor en cualquiera de las 6 M's tiene un buen potencial para el éxito como servicio móvil. Si la evaluación de las 6 M's del servicio es insatisfactoria, se debe rediseñar el servicio fortaleciendo los atributos mencionados.

\section{Entrega}

Terminada la depuración de la aplicación y atendidos todos los requerimientos de última hora del cliente se da por finalizada la aplicación y se procede a la entrega del ejecutable, el código fuente, la documentación y el manual del sistema.

- Manuales: el objetivo es el entrenamiento; una aplicación móvil debe constar de un manual del sistema donde se indique el proceso de instalación, la atención a posibles fallas en el tiempo de ejecución y, las especificaciones técnicas mínimas de hardware y software que requiere el equipo, para el funcionamiento adecuado del aplicativo desarrollado.

- Distribución: se define el canal de comercialización de la aplicación, con el propósito de adecuar la aplicación al medio de distribución. A continuación se mencionan algunos de los canales de distribución existentes.

Las tiendas físicas u outlets, especializadas o no, corresponden a las tiendas que venden dispositivos y servicios de telecomunicaciones, normalmente operadores o marcas como Apple.

Los portales de operadores o desarrolladores de servicios, ofrecen un catálogo amplio de aplicaciones y ventas vía Web Site desde el PC, que luego son instaladas en el móvil. 


\section{investigación}

Las Applications Stores, son las tiendas online de los fabricantes de dispositivos o de sistemas operativos.

OTA, Over the Air, es la comercialización de aplicaciones a través de la interfaz de radio. Los contenidos móviles son distribuidos a los terminales usando SMS, WAP y Streaming, entre otros.

Los servicios basados en el Subscriber Identity Module (SIM), o la Universal Integrated Circuit Card (UICC), son aplicaciones instaladas previamente en el chip de estas tarjetas.

\section{RESULTADOS}

El método propuesto, llamado DrMovil, se utilizó para desarrollar el servicio de $m$-Health para prevenir y monitorizar pacientes con enfermedades relacionadas a la glucosa y tensión arterial (HTA). DrMovil se desarrolló considerando las tecnologías presente en Latinoamérica en un tiempo de cuatro meses, cumpliendo el Time-to-Market y obteniendo una evaluación de éxito satisfactoria por parte de un grupo de usuarios y expertos; los desarrolladores del servicio son: Miguel Jose Rivera Ospino y Andrés Fabián Díaz-Granados Tobías estudiantes de Ingeniería, y el docente Luis Leonardo Camargo Ariza. En la figura 3 se muestra la ejecución de cada fase con la dedicación del tiempo en semanas.

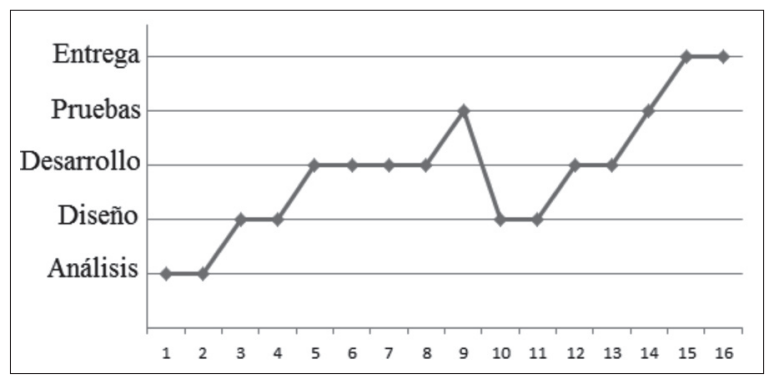

Figura 3. Tiempos de ejecucion del servicio DrMovil

Fuente: elaboración propia.
El servicio desarrollado está soportado por: DrMovil.apk, aplicación para usuarios de móviles con S.O. Android; DrMovil.jar, aplicación para usuarios de móviles con S.O. Symbia y OS7 de BlackBerry; y DrMovilServidor, software que incorpora la lógica del servicio y la aplicación Web dirigida al médico supervisor del servicio. DrMovilServidor y DrMovil fueron diseñados para ejecutarse en conjunto como se ilustra en la figura 4.

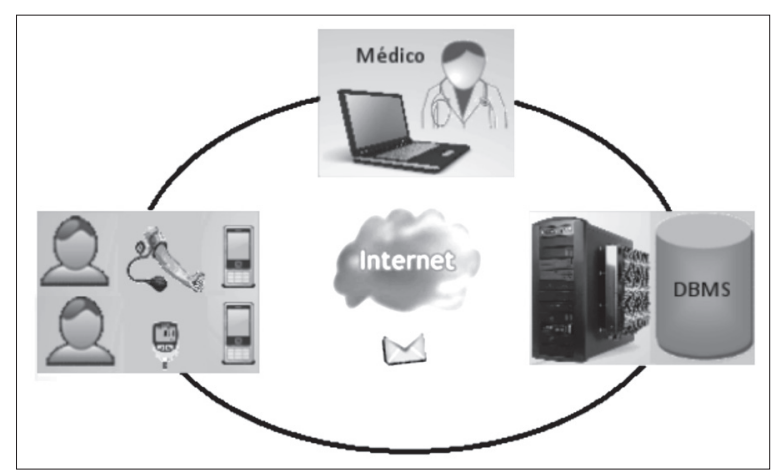

Figura 4. Descripción del servicio DrMovil

Fuente: elaboración propia.

DrMovil.apk y DrMovil.jar están dirigidas a personas: hipertensas, diabéticos, hipoglucémicos, deportistas, mujeres embarazadas, entre otras, permitiendo al usuario utilizar su dispositivo móvil para:

- Ingresar y almacenar los valores de glucosa, tensión arterial sistólica (PAS) y tensión arterial diastólica (PAD) en la memoria interna del dispositivo.

- Comparar los valores ingresados con los estándares mundiales de las mediciones de estas variables fisiológicas.

Para la HTA se clasifican las medidas en: Normal, Pre-hipertensión, HTA1, HTA2, como se muestra en la tabla 1 (U.S. Department of health and human services, 2004). 


\section{investigación}

Tabla 1. Clasificación de la hipertensión arterial

\begin{tabular}{|c|c|c|}
\hline Categoría & PAS & PAD \\
\hline Normal & $<120$ & $<80$ \\
\hline Pre-hipertensión & $120-139$ & $80-89$ \\
\hline HTA estado 1 & $140-159$ & $90-99$ \\
\hline HTA estado 2 & $\geq 160$ & $\geq 100$ \\
\hline
\end{tabular}

Fuente: elaboración propia.

Los niveles de glucosa en $\mathrm{mg} / \mathrm{dl}$ se clasificaron en bajo $(<80)$, normal $(>80$ y $<155)$ y alto $(>155)$, teniendo en cuenta la tabla 2 (Alfaro, Simal y Botella, 2000).

Tabla 2. Criterios de control para la diabetes mellitus

\begin{tabular}{|c|c|c|c|}
\hline Categoría & Bueno & Límite & Malo \\
\hline Glucemia basal $(\mathrm{mg} / \mathrm{dl})$ & $80-100$ & $<140$ & $>140$ \\
\hline $\begin{array}{c}\text { Glucemia después de } \\
\text { comer }(\mathrm{mg} / \mathrm{dl})\end{array}$ & $80-144$ & $<180$ & $>180$ \\
\hline $\begin{array}{c}\text { Glucemia antes de } \\
\text { comer }(\mathrm{mg} / \mathrm{dl})\end{array}$ & $100-140$ & $<120$ & $>140$ \\
& & & $<100$ \\
\hline
\end{tabular}

Fuente: elaboración propia.

- Visualizar de forma gráfica la evolución histórica de los valores de las mediciones almacenadas y enviar la información sobre las mediciones realizadas a una base de datos en internet para ser analizada por el médico.

- Enviar un mensaje de texto corto SMS con la información de la medida corporal al teléfono móvil del médico de forma automática.

DrMovilServidor está dirigido a las empresas prestadoras de servicios de salud que quieran utilizar los beneficios de las tecnologías de información y comunicación para mejorar los procesos de supervisión de pacientes y prevención de enfermedades. Las funciones básicas del DrMovilServidor son:

- Recibir, procesar y almacenar, en la base de datos, los valores de las medidas fisiológicas de: glucosa, tensión arterial sistólica y tensión arterial diastólica; enviadas desde los dispositivos móviles del usuario.

- Permitir a los médicos del sistema acceder a la información de las medidas fisiológicas de sus pacientes, desde la Web por medio de la autenticación del usuario.

- Permitirles a los médicos del sistema, enviar desde la web, el diagnóstico al teléfono móvil del paciente, por medio de un mensaje de texto SMS, según la evolución de las medidas.

- Permitirle al administrador del sistema crear y editar el perfil de los médicos del sistema, asignar y modificar la lista de pacientes que atiende cada médico adscrito al sistema.

Las aplicaciones se instalaron y probaron en varios dispositivos comerciales con soporte para J2ME y sistema operativo Android, comprobando los parámetros de: presentación, navegación, ayudas, gráficas, almacenamiento y sincronización con el servidor. En la figura 5 se muestran algunos pantallazos de la ejecución de la aplicación DrMovil.jar y DrMovil.apk. En la figura 6 se muestra algunos pantallazos de la aplicación web.

Posteriormente se realizó una jornada de utilización del sistema DrMovil en el campus de la Universidad del Magdalena; en esta jornada algunos estudiantes se tomaron muestras de glucosa o presión arterial, acompañados de estudiantes de enfermería de últimos semestres, utilizando el DrMovil instalado en sus teléfonos personales para registrar, comparar y enviar la información de las medidas tomadas; además, los próximos profesionales de enfermería realizaron pruebas de funcionamiento a la aplicación Web DrMovilServidor, revisando las muestras almacenadas por los voluntarios, ver figura 7 . 


\section{investigación}

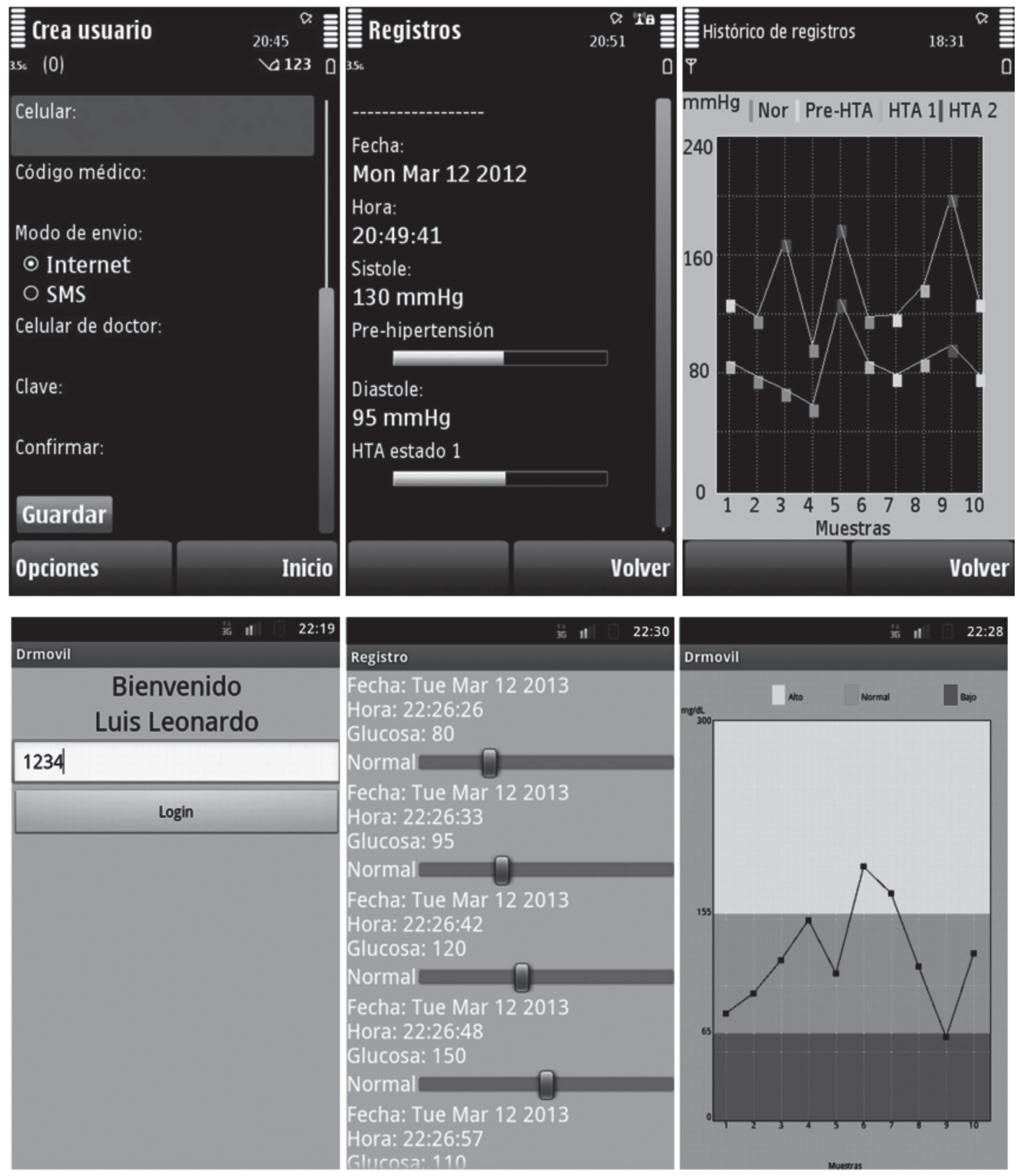

Figura 5. DrMovil en dispositivos comerciales

Fuente: elaboración propia. 


\section{investigación}


Figura 6. Algunas pantallas de la aplicación Web

Fuente: elaboración propia.
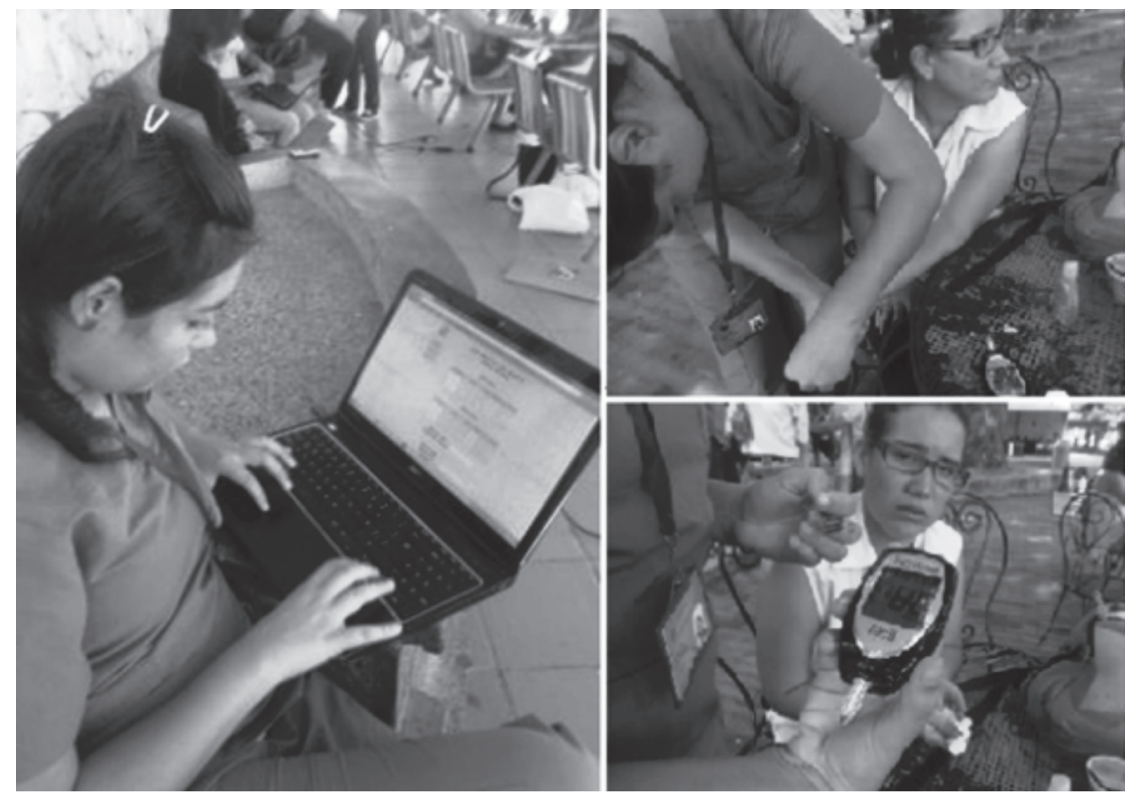

Figura 7. Prueba de DrMovil con usuarios

Fuente: elaboración propia. 


\section{investigación}

Se les solicitó a los usuarios mencionados y algunos expertos, diligenciar una encuesta sobre el servicio. Las características de la muestra de los usuarios se enmarcan en la figura 8 .

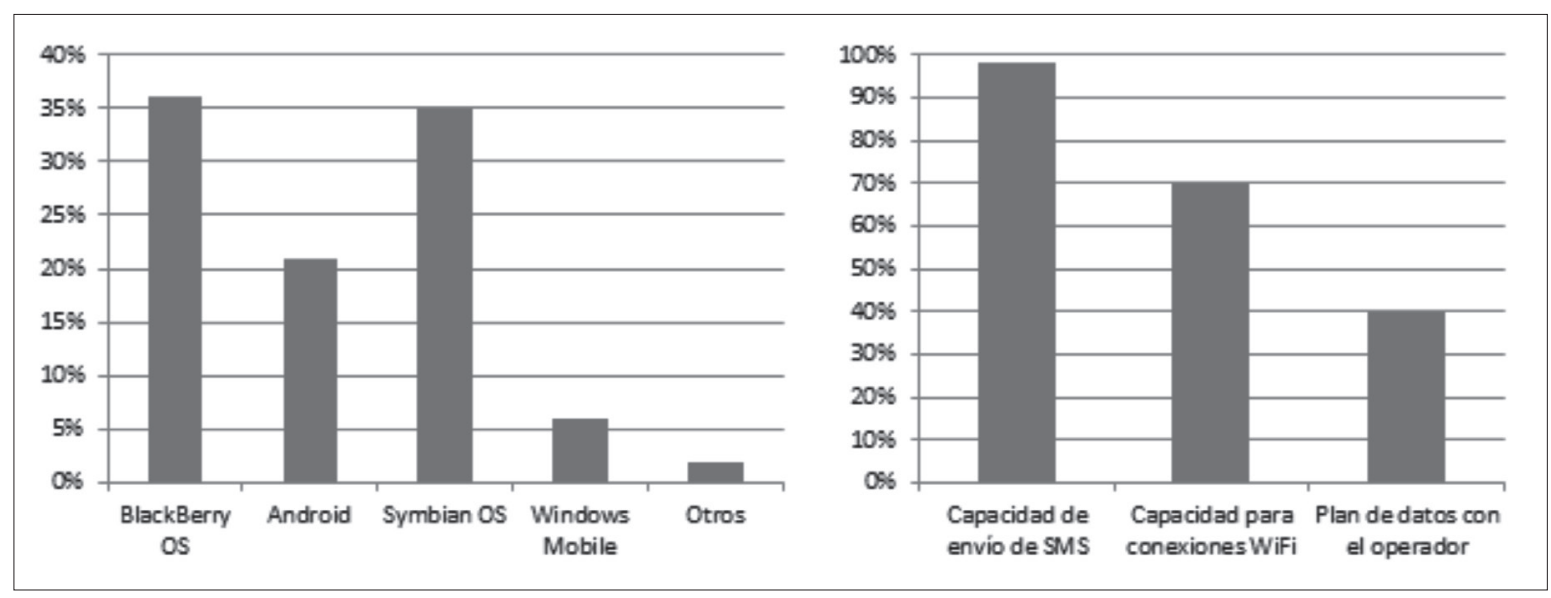

Figura 8. Caracteristicas de los usuarios que evaluaron el servicios

Fuente: elaboración propia.

Los usuarios con sistema operativo Windows Mobile y otros no pudieron instalar, ni evaluar el servicio. Los resultados de la encuesta se utiliza- ron para valorar el potencial de éxito del servicio, la evaluación se sintetiza en la tabla 3.

Tabla 3. Evaluación de las 6 M's del servicio DrMovil

\begin{tabular}{|c|c|c|c|}
\hline Atributo & Definición & Calificación & Justificación \\
\hline Momento & $\begin{array}{l}\text { Un servicio que cuente con este atribu- } \\
\text { to debe estar disponible en cualquier } \\
\text { instante de tiempo en que el usuario } \\
\text { desee usar dicho servicio. }\end{array}$ & 4 & $\begin{array}{l}\text { El paciente puede ingresar la medida en cualquier } \\
\text { momento, pero la respuesta del diagnóstico depende } \\
\text { del horario de atención del médico. }\end{array}$ \\
\hline Movilidad & $\begin{array}{l}\text { Un servicio móvil debe ser "móvil" por } \\
\text { naturaleza, la ubicación debe ser una } \\
\text { parte integral del servicio. }\end{array}$ & 4 & $\begin{array}{l}\text { El usuario se puede desplazar a cualquier lugar y rea- } \\
\text { lizar sus registros, siempre y cuando tenga cobertura } \\
\text { del operador. La atención médica se ajusta al área de } \\
\text { cobertura del operador. }\end{array}$ \\
\hline Dinero & $\begin{array}{l}\text { Como cualquier acción comercial, un } \\
\text { servicio móvil tiene un fin lucrativo, ya } \\
\text { sea para el operador, para el proveedor } \\
\text { del servicio o para el usuario. }\end{array}$ & 5 & $\begin{array}{l}\text { Aunque la aplicación es de descarga gratuita, ésta } \\
\text { genera ingresos al operador al ocasionar tráfico en } \\
\text { la red de datos. } \\
\text { La entidad prestadora de servicios de salud (EPS) } \\
\text { reduce costos por la atención de pacientes, lo que } \\
\text { permite redirigir los ingresos a casos de mayor rele- } \\
\text { vancia. } \\
\text { El paciente reduce los costos de traslado a la EPS. }\end{array}$ \\
\hline
\end{tabular}




\section{investigación}

\begin{tabular}{|l|l|l|l|}
\hline Atributo & \multicolumn{1}{|c|}{ Definición } & Calificación & \multicolumn{1}{c|}{ Justificación } \\
\hline Yo & $\begin{array}{l}\text { Se refiere al nivel de personalización de } \\
\text { un servicio. }\end{array}$ & 3 & $\begin{array}{l}\text { El servicio presenta un grado de personalización por- } \\
\text { que permite que el usuario seleccione: el médico, el } \\
\text { modo de ver los registros almacenados y el tipo de } \\
\text { conexión para enviar los datos. }\end{array}$ \\
\hline Máquina & $\begin{array}{l}\text { La tecnología (terminal o redes) siem- } \\
\text { pre es el factor que posibilita o limi- } \\
\text { ta; el atributo máquina busca añadir } \\
\text { potencia a los dispositivos de última } \\
\text { generación que cada vez tienen mayo- } \\
\text { res prestaciones a nivel de hardware y } \\
\text { software. }\end{array}$ & 4 & $\begin{array}{l}\text { El servicio solo puede ser soportado por celulares de } \\
\text { gama media que posean Java o Android, y conexión } \\
\text { a la red de datos. } \\
\text { El usuario puede hacer un buen uso de la aplicación } \\
\text { sin importar la resolución de la pantalla y el tipo del } \\
\text { teclado. }\end{array}$ \\
\hline Multiusuario & $\begin{array}{l}\text { Busca extenderse dentro de la comu- } \\
\text { nidad, que el servicio sea interactivo } \\
\text { y que pueda utilizarse por múltiples } \\
\text { usuarios de manera simultánea. }\end{array}$ & 1 & $\begin{array}{l}\text { Aunque la aplicación permite una comunicación en } \\
\text { dos direcciones, no permite interactuar con la perso- } \\
\text { na en el otro extremo del sistema. }\end{array}$ \\
\hline
\end{tabular}

Fuente: elaboración propia.

\section{CONCLUSIONES}

Las aplicaciones móviles deben considerar las características del entorno de ejecución de la aplicación que dispone el teléfono, para garantizar el correcto funcionamiento de la misma.

Las aplicaciones móviles pueden ayudar a solventar los problemas de tipo particular o general de la sociedad, debido a sus características de movilidad y ubicuidad.

Los entornos integrados de desarrollo (IDE) facilitan la elaboración de aplicaciones móviles, porque ofrecen herramientas de edición, compilación, depuración, análisis y ejecución en una misma interfaz, mejorando los tiempos de ejecución de las etapas de diseño, desarrollo y pruebas, de la metodología propuesta.

J2ME es una plataforma soportada por una gran variedad de dispositivos, incluyendo aquellos que no poseen alta capacidad de procesamiento, como los teléfonos móviles no inteligentes.

Android, permite la creación de formatos de interfaz más amigables para el usuario, sin gran complejidad en el código, por la característica de edición mediante archivos xml.

La utilización de SMS para mensajes emergentes permite que el usuario reciba la información sin necesidad de tener activa la aplicación, mejorando el rendimiento computacional y el tiempo útil de la batería.

El uso de aplicativos m-Health mejora los tiempos y los costos que implican el diagnóstico de pacientes con enfermedades que requieran un control periódico, porque permite conectar al médico y al paciente, sin importar su ubicación geográfica.

El servicio desarrollado facilita el autocontrol a pacientes con problemas de azúcar e hipertensión, porque guarda y grafica los históricos de las 


\section{investigación}

medidas corporales, referenciando éstas con valores de estándares mundiales de prevención.

En el tiempo que se realizó la aplicación, la tendencia de la programación en dispositivos móviles migró hacia los nuevos sistemas operativos Android y Windows Phone.

\section{FINANCIAMIENTO}

Este artículo es un producto del proyecto de investigación: Sistema de interacción entre un paciente y el médico utilizando las tecnologías móviles, financiado por la Universidad del Magdalena.

\section{REFERENCIAS}

Agusti, R., Bernardo, F., Casadevall, F., Ferrús, R., Pérez, J. y Sallent, O. (2010). LTE: Nuevas Tendencias en Comunicaciones Móviles. Madrid: Fundación Vodafone España.

Ahonen, T., Barret, J. \& Golding, P. (2002). Services for UMTS, Creating Killer Applications in 3G. West Sussex: John Wiley \& Sons.

Alfaro, J., Simal, A. y Botella, F. (2000). Tratamiento de la diabetes mellitus. Revista Información Terapéutica del Sistema Nacional de Salud, 24(2), 33-43.

Autelsi, Asociación española de usuarios de telecomunicaciones y de la sociedad de la información (2009). Modelos de negocios en dispositivos y aplicaciones móviles. Recuperado de http://www.autelsi.es/cms/autel/ images/socios/aplicmov.pdf.

Beck, K., Beedle, M., Bennekum, A., Cockburn, A., Cunningham, W., Fowler, M. \& Thomas, D. (2001). Manifesto for Agile Software Development. Utah: The Agile Alliance. Available http://agilemanifesto.org/.

Gómez, R., Galvis, A. y Mariño, O. (1998). Ingeniería de software educativo con modelaje orientado por objetos: un medio para desarrollar micromundos interactivos. Informática Educativa UNIANDES - LIDIE, 11(1), 9-30.
Halonen, T., Romero, J. \& Melero, J. (2003). Gsm, Gprs And Edge Performance Evolution Towards $3 G$ Umts. Inglaterra: John Wiley \& Sons.

Holma, H. \& Toskala, A. (2004). WCDMA for UMTS. Radio Access For Third Generation Mobile Communications. Inglaterra: John Wiley \& Sons.

Istepanian, R., Jovanov, E. \& Zhang, Y. (2004). Guest editorial introduction to the special section on m-health: Beyond seamless mobility for global wireless healthcare connectivity. IEEE Transactions on information technology in biomedicine, 8(4), 405-412.

Kylmäkoski, R. (2005). RaPiD7: A Collaborative Method for the Planning Activities in Software Engineering - Industrial Experiment. Recuperdado de Information Technology for European Advancement: http://www. agile-itea.org/public/papers/RaPiD7.pdf.

Kynkäänniemi, T. \& Komulainen, K. (2006). Agile Documentation in Mobile-D Projects (Agile Deliverable D.2.10). Recuperado de Information Technology for European Advancement: http://www.agile-itea.org/ public/deliverables/ITEA-AGILE-D2.10 v1.0.pdf. 
Montilla, A. (2004). Desarrollo de servicios para telefonía móvil 2.5g y $3 g$. Caracas: CONATEL/CEDITEL.

Rahimian, V. \& Ramsin, R. (2008, 6 de junio). Designing and agile methodology for mobile software development: a hybrid ethod engineering approach. Second International Conference on Research Challenges in Information Science. Recuperado de http://ieeexplore.ieee.org/xpl/ articleDetails.jsp?arnumber $=4632123 \& \mathrm{pu}$ number\%3D4620134\%26sortType\%3Das c_p_Sequence $\% 26$ filter\%3DAND $\% 28 p$ IS_Number\%3A4632084\%29\%26pageNu mber\%3D2.

UIT, Unión Internacional de Telecomunicaciones. (2012). World Telecommunication ICT Indicators Database, Mobile cellular

\section{investigación}

subscriptions. Recuperado de http://www. itu.int/ITU-D/ict/statistics/material/excel/ Mobile-cellular2000-2011.xls.

U.S. Department of health and human services. (2004). Seventh Report of the Joint National Committee on Prevention, Detection, Evaluation, and Treatment of High Blood Pressure. Recuperado de http:// www.nhlbi.nih.gov/guidelines/hypertension/jnc7full.pdf.

Vital Wave Consulting. (2009). MHealth for Development: The Opportunity of Mobile Technology for Healthcare in the Developing World. Recuperado de http://www. globalproblems-globalsolutions-files.org/ unf website/assets/publications/technology/mhealth/mHealth_for_Development_ full.pdf 